\title{
Phoenix: Umgestaltung eines Industriestandortes in einen Wohn- und Gewerbepark mit wasserwirtschaftlichen Ansprüchen
}

\author{
Christoph Schöpfer • Ulrich Krath · Kerstin Kowtsch
}

Eingegangen: 19. Juli 2010/Akzeptiert: 12. September 2010/Online veröffentlicht: 12. Oktober 2010

(C) Springer-Verlag 2010

\begin{abstract}
Zusammenfassung Die Umwandlung vom ehemaligen Hochofenstandort „Hermannhütte“ in die Gewerbe-, Wohnund Dienstleistungsparks PHOENIX West und PHOENIX Ost in Dortmund-Hörde ist mit einigen wasserwirtschaftlichen Herausforderungen verbunden. Der Rückhalt von Sedimenten und Nährstoffen spielt dabei eine wesentliche Rolle. Zur Erreichung der gesetzten Ziele kommen verschiedene Bewirtschaftungsmaßnahmen zum Einsatz. Die Anlage eines künstlichen grund- und regenwassergespeisten Sees in PHOENIX Ost stellt andere Anforderungen an die Bewirtschaftung als die konventionelle Reinigung und Rückhaltung des Oberflächenwassers in PHOENIX West. Sowohl konventionelle Verfahren wie Regenklär- und Rückhaltebecken als auch Bodenfilteranlagen, Phosphateliminierung, die Biodyozon ${ }^{\circledR}$-Anlage und Biomassenmanagement sorgen für eine gute Wasserqualität.
\end{abstract}

Schlüsselwörter Bodenfilter $\cdot$ Regenwasserbewirtschaftung $\cdot$ Sedimentrückhalt $\cdot$ Umwandlung

Transformation of an industrial site into residential and commercial parks including challenges in water management

Abstract The transformation of the former blast-furnace site "Hermannhütte" in Dortmund-Hoerde into commercial, residential and service parks, called PHOENIX East

Verantwortliche Herausgeber: Organisationskomitee der Tagung

C. Schöpfer $(\bowtie) \cdot U$. Krath $\cdot$ K. Kowtsch

Björnsen Beratende Ingenieure $\mathrm{GmbH}$,

Maria Trost 3, 56070 Koblenz, Deutschland

E-Mail: info@bjoernsen.de and PHOENIX West involves some unusual challenges concerning the water management. The retention of sediments and nutrients plays an important role. To achieve the objectives, various management measures are being used. The installation of an artificial lake in PHOENIX East, which is groundwater- and rainfed, has different requirements than the conventional purification and retention of surface water in PHOENIX West. Both conventional methods such as storm water sedimentation tank and -reservoirs as well as soil filtration, phosphates elimination, Biodyozon $^{\circledR}$-plant and biomass management ensure good water quality.

Keywords Conversion - Retention of sediments - Soil filtration $\cdot$ Storm water management

\section{Einleitung}

Nach mehr als 150 Jahren wurde im Jahr 2001 das Stahlwerk Hermannshütte im Dortmunder Stadtteil Hörde stillgelegt. Die verfahrenstechnischen Anlagen wurden demontiert und nach China zur Neuerrichtung verkauft. Bis Ende 2004 wurde ein Großteil der oberirdischen Bausubstanz abgebrochen.

Die gesamte, rd. 215 ha umfassende Industriebrache, gegliedert in die Standorte PHOENIX West und PHOENIX Ost, soll als integriertes Gesamtprojekt revitalisiert werden (Abb. 1). Der Schwerpunkt liegt beim Standort PHOENIX West auf der Ansiedlung von Gewerbebetrieben der Mikrotechnologie und der Logistik. Der Standort PHOENIX Ost bietet in Ergänzung hierzu ein hochwertiges Wohn- und Freizeitangebot und zielt auf die Etablierung von Dienstleistungsunternehmen der Informations- und Kommunikationswirtschaft ab. 


\section{Zukunftsstandort PHOENIX}

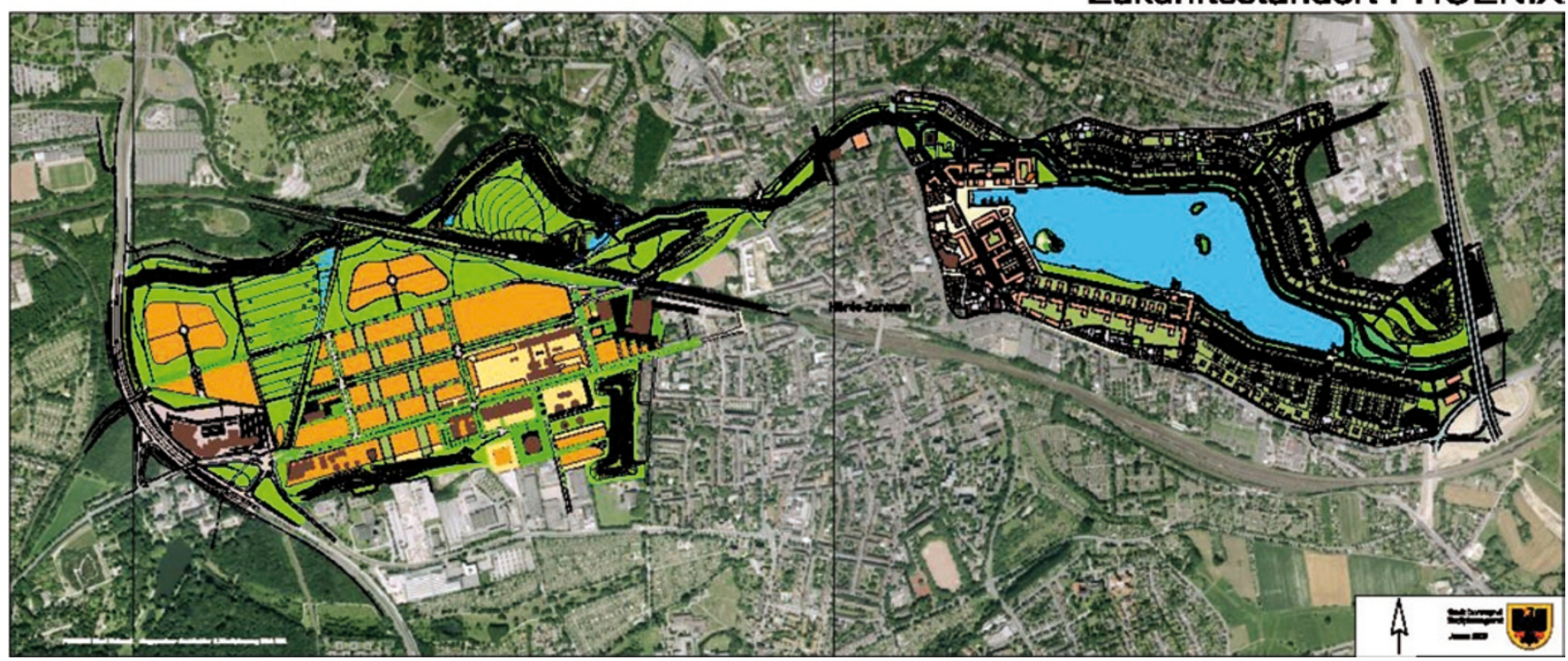

Abb. 1 Standort PHOENIX West und Ost

\section{PHOENIX Ost}

Im Mittelpunkt des Gebietes PHOENIX Ost steht der PHOENIX See (Abb. 1) und die renaturierte Emscher. Der PHOENIX See umfasst rd. 24 ha Fläche im rd. 100 ha großen Standort PHOENIX Ost und wird als Flachwassersee mit einer Wassertiefe von rd. 3,0 m angelegt. Er ist grundwassergespeist und dient der Regenwasserbewirtschaftung durch Aufnahme der unbelasteten Dachflächenwässer der zukünftig umliegenden Bebauung. Nördlich des Sees wird die derzeit noch im Werksgelände verrohrte Emscher in einer Auenlandschaft offengelegt und renaturiert.

\subsection{Seewasserbewirtschaftung}

Neben der Naherholungsfunktion dient der PHOENIX See der Schaffung von rd. $240000 \mathrm{~m}^{3}$ Hochwasserrückhalteraum für die Emscher als Teil eines übergeordneten Steuerungskonzeptes verschiedener Hochwasserschutzeinrichtungen im Oberlauf der Emscher. Aus Gründen der Seewassergüte verläuft die Emscher im Nebenschluss.

Der Flachwassersee mit rd. 3,0 m Tiefe und einem Einstauvolumen von rd. $600000 \mathrm{~m}^{3}$ ist in Bezug auf seine Höhenlage so angeordnet, dass im Dauerstau in der gesamten Osthälfte Grundwasser zuströmt. Um die Wasserverluste über niedrigere Grundwasserstände nach Westen hin einzuschränken, wird ein Teil der Seesohle mit bindigen Böden gegen Ufereinfassungen abgedichtet. Zum Ausgleich von saisonalen Seewasserverlusten kann sowohl von der Emscher als auch vom benachbarten Hörder Bach Wasser zugespeist werden.

Ein besonderes Augenmerk gilt der Gewässergüte des PHOENIX Sees. Leitbild ist hierbei gemäß potenziell na- türlichem Zustand ein schwach eutrophes Gewässer mit rd. $50 \mathrm{mg} / \mathrm{m}^{3}$ Gesamtphosphorgehalt. Um diesen Zustand zu erreichen, muss möglichst wenig Nährstoff eingetragen werden sowie eine gute Belüftung und Sauerstoffversorgung des Wassers, ein gezielter Nährstoffentzug und eine vielfältige stabile Seeflora und Seefauna etabliert werden.

Die gute Belüftung des Seewassers wird durch die geringe Wassertiefe und die Seeausrichtung in West-Ost-Richtung erreicht. Die Minimierung des Nährstoffeintrages erfolgt durch Speisung mit nährstoffarmem Grundwasser und ausschließlich der Regelzuleitung von Dachflächenwasser. Der gezielte Nährstoffentzug wird einerseits durch Biomassebewirtschaftung (Mähen und Entnahme von Makrophyten) und andererseits durch eine parallel betriebene Bodenfilteranlage sowie eine Phosphateliminationsanlage erreicht. Das aufbereitete Seewasser wird in die Emscher geleitet bzw. rezirkulierend in den See geführt. Eine wesentliche Funktion des Bodenfilters ist die Verhinderung der Vermischung von Biozönosen des PHOENIX-Seewassers und der Emscher. Im Rezirkulationsbetrieb werden Nährstoffe und Feinpartikel aus dem Seewasser entfernt. Die Phosphatentfernung aus dem Seewasser erfolgt durch Adsorptionsprozesse von Phosphaten an Eisenhydroxidgranulat. Der Phosphateliminationsanlage wird zur Verhinderung bzw. Minimierung von Kolmationserscheinungen des Eisenhydroxidgranulats ein Membrantrommelfilter vorgeschaltet. Über die mit Filtertuch bespannten Filterscheiben werden partikuläre Stoffe einschließlich Schwebstoffe entfernt.

Zur Initiierung einer stabilen Seeflora und Seefauna werden im Zuge der Flutung Makrophyten auf die Seesohle gesetzt, spezielle Flachwasserzonen mit Schilf angelegt und ein Fischbesatz nach Maßgabe eines Hegeplanes umgesetzt. 


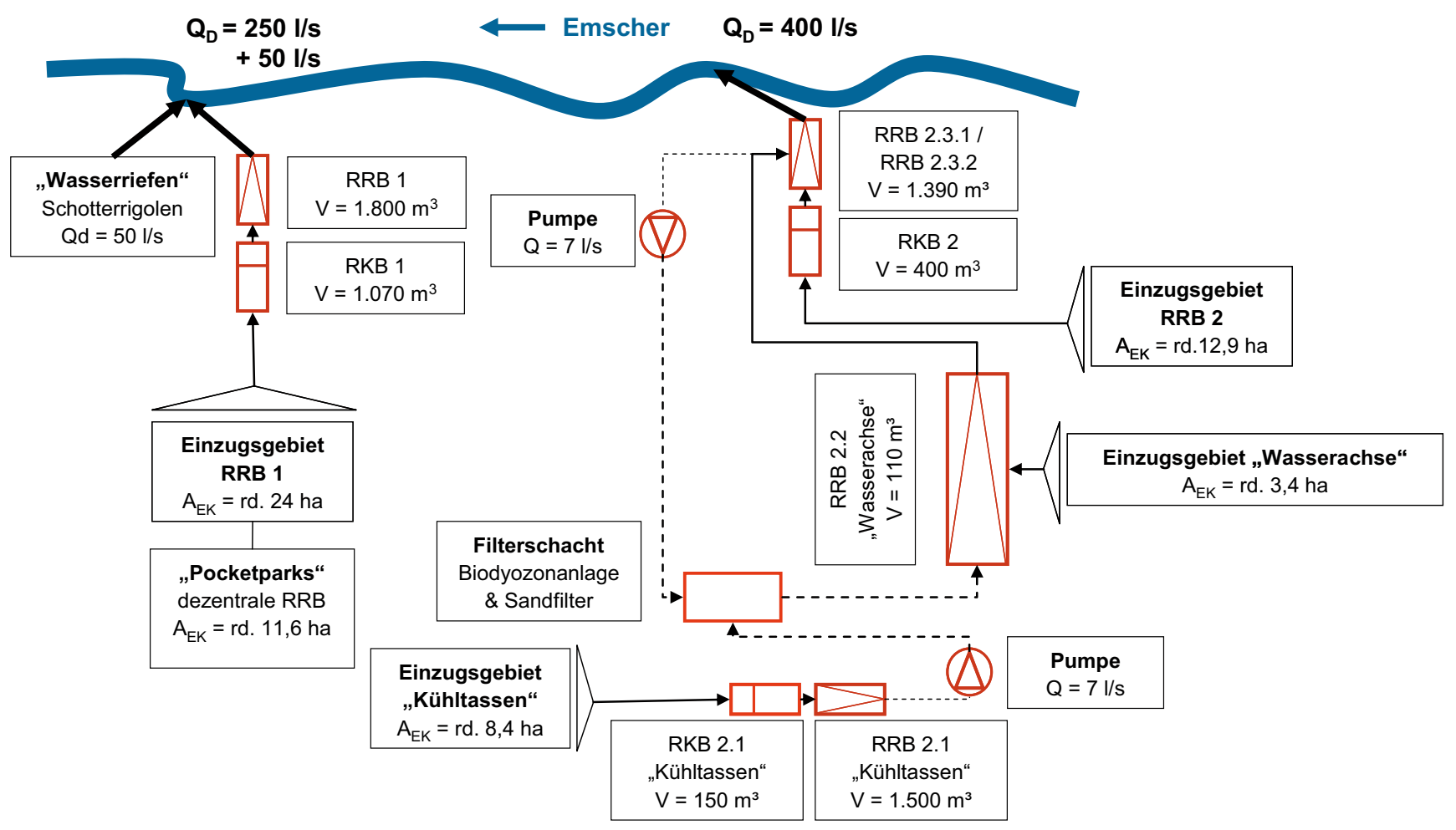

Abb. 2 Verfahrensschema Regenwasserbewirtschaftung PHOENIX West

\subsection{Ausgestaltung von See und Emscher}

Neben den rein wasserwirtschaftlichen Ansprüchen sind im Hinblick auf das gewünschte hochwertige Wohn- und Freizeitangebot eine Vielzahl von Infrastruktureinrichtungen vorgesehen. Wesentliches Merkmal ist ein umfassendes Wegenetz entlang von See und Emscher mit insgesamt drei Fuß- und Radwegebrücken über die Emscher. Die Wehranlage wird ebenfalls als Querungselement der Emscher genutzt und mit einer speziellen Aussichtsplattform in Szene gesetzt.

Auf der Westseite des Sees entsteht ein neues Zentrum von Hörde mit Hafen, Slipanlage und Stegen für Segelboote, Promenaden, Plätzen und Gastronomie. Eine besondere Attraktivität entsteht durch das ufernahe Anlegen einer Insel für u. a. kulturelle Veranstaltungen mit Zugangsbrücke.

Der Osten des Sees ist demgegenüber prioritär der Ökologie gewidmet mit kleinen, naturnahen Aufenthaltsbereichen und einer Steganlage mit gehobener Kleingastronomie. Zwei Landschaftsinseln gliedern in diesem Bereich den See.

Im Süden wird ein Sport- und Rettungsstützpunkt angelegt, ebenfalls mit Slip- sowie Steganlagen für Ruder- und Segelboote. Hinzu kommen Holzplattformen am Wasser und Kleinspielfelder.

Die Phosphateliminationsanlage wird im Nordwesten realisiert und erfüllt neben der Seewasseraufbereitung besondere architektonische Ansprüche.

\subsection{Bauliche Realisierung}

Die Arbeiten für die wasserbaulichen Ausbaugewerke begannen im April 2009 und werden im Wesentlichen Ende des Jahres 2010 abgeschlossen sein, dem beabsichtigten Flutungsbeginn. Die Seeflutung selbst wird voraussichtlich 1 bis 1,5 Jahre dauern.

Parallel werden seit Juli 2009 die Erschließungsarbeiten der zukünftigen städtebaulichen Flächen, betreffend Verund Entsorgung sowie Straßenbau, durchgeführt.

\section{PHOENIX West}

Der Mikro- und Nanotechnologiestandort PHOENIX West besteht im Wesentlichen aus dem Gewerbe- und Technologiepark und einem rd. 60 ha großen Landschaftspark, dem PHOENIX-Park. Die Erschließung des ehemaligen Hochofenstandortes wurde nach der Bodensanierung im Zeitraum von 2007 bis zum Sommer 2009 realisiert.

Das gesamte Gebiet wurde im Trennsystem erschlossen. Das Schmutzwasser ist an das städtische Kanalnetz angeschlossen worden, das Regenwasser wird über verschiedene Regenwasserbewirtschaftungsmaßnahmen an zwei Einleitungsstellen in die Emscher geleitet (Abb. 2).

Um die Emscher vor Stoffeinträgen und Abflussspitzen zu schützen, die durch die Bebauung und Nutzung des Ge- 
werbeparks entstehen, wurden sowohl Reinigungs- als auch Rückhaltemaßnahmen getroffen.

Zum Rückhalt von Sedimenten, Schadstoffen, Leichtflüssigkeiten und Schwimmstoffen wurden klassische dauergestaute Regenklärbecken errichtet. Durch bauliche Maßnahmen, wie Tauchwände, werden Schwimmstoffe und Leichtflüssigkeiten zurückgehalten. Schwerere Sedimente setzen sich auf der Beckensohle ab. Über den Klärüberlauf wird das gereinigte Oberflächenwasser bis zum Erreichen des kritischen Abflusses und darüber hinaus über das Trennbauwerk in eines der Regenrückhaltebecken geleitet. Die Reinigung der Regenklärbecken wird durch sogenannte Schwallspültrommeln vorgenommen. Die Trommeln sind im Becken im Dauerstaubereich aufgehängt und werden im Normalbetrieb über den Dauerwasserspiegel mit Oberflächenwasser gefüllt. Zur Reinigung des Beckens wird der Inhalt des Regenklärbeckens in den Schmutzwasserkanal entleert. Durch das Absinken des Wasserspiegels im Pumpensumpf wird die Spültrommel über einen mechanischen Schwimmkörper ausgelöst. Der erzeugte Spülschwall befördert die abgelagerten Sedimente in den Pumpensumpf. Das dort zurückbleibende Schlammwasser wird durch einen Saugwagen entnommen.

In den Regenrückhaltebecken wird der Ablauf in die Emscher über eine mechanische, wasserstandsabhängige Drossel reduziert und erzeugt folglich einen Einstau der $\mathrm{Zu}-$ laufwassermengen in den Rückhalteräumen. Es wurden verschiedene Arten von Retentionsmöglichkeiten zum Einsatz gebracht, u. a. naturnahe Erdbecken, umgewandelte ehemalige Industrieanlagen, die sogenannten Kühltassen, Rigolensysteme im „Landschaftskeil“, dezentrale „Pocketparks“ sowie die im Rahmen der Landschaftsgestaltung errichtete „Wasserachse“, die aus 10 offenen Stahlbetonkaskaden besteht.

Zur Verhinderung von Algenbildung in den Sommermonaten, wird in der „Wasserachse“ ein konstanter Durchfluss von 7 1/s gewährleistet. Der Zulauf wird über die Rückhaltebecken „Kühltassen“ bzw. dem dauergestauten RRB „Brückental“" zur Verfügung gestellt. Diesem Wasser wird über eine sogenannte Biodyozon ${ }^{\circledR}$-Anlage erzeugtes Desinfektionsmittel beigefügt, das durch sein hohes Redoxpotenzial Mikroorganismen und Algen abtötet. Gleichzeitig wird über die Biodyozon ${ }^{\circledR}$-Anlage Sauerstoff in die Kaskaden eingetragen. Fäulnisprozesse werden damit in den dauergefüllten Kaskaden unterbunden und das Ansetzen von Grünbelag verhindert.

Alle Anlagen der Regenwasserbewirtschaftung mussten zum Untergrund hin abgedichtet werden, um Rücklösungsprozesse von schadstoffbelasteten Böden zu vermeiden.
4 Erfahrungen mit bestehenden Anlagen der Regenwasserbewirtschaftung am Beispiel Nordeifel Aufbereitung von Mischwasserentlastungen und Regenwassereinleitungen im Einzugsgebiet von Trinkwassertalsperren

Im Auftrag der Wassergewinnungs- und Wasseraufbereitungsgesellschaft Nordeifel (WAG) wurde anhand von Pilotanlagen untersucht, wie mikrobielle und anorganische Belastungen von Mischwasser- und Regenwasserableitungen aus urbanen Gebieten im Einzugsgebiet von Trinkwassertalsperren verringert werden können. Im Mischsystem wurden für die Entlastungsabflüsse vertikale Retentionsbodenfilter errichtet.

Im Trennsystem sollte durch vier Pilotanlagen untersucht werden, wie der hydraulische als auch stoffliche Rückhalt bzw. Abbau funktioniert. Es wurden jeweils zwei horizontale Bodenfilter (Abb. 3) mit vorgeschaltetem Retentionsbecken und zwei vertikale Retentionsbodenfilter als Versuchsanlagen aufgebaut. Bei allen Anlagen wurden ein Geröllfang und ein Sandfang im Zulaufbereich installiert. Im Zeitraum von 2001 bis 2003 wurden verschiedene Parameter wie Abflussganglinien, TOC, Ammonium und Nitrat, Phosphor, Chlorid- und Leitfähigkeit, Coliforme und E.-Colibakterien analysiert und ausgewertet.

Sowohl horizontale als auch vertikale Bodenfilteranlagen entsprechen den Anforderungen an die Regenwasserbehandlung ,naturnaher Bauart“. Es konnte bei beiden Anlagentypen nachgewiesen werden, dass die Zulaufwellen durch die Retentionsvolumina gedämpft werden. Der stoffliche Rückhalt bzw. Abbau konnte ebenfalls in allen Versuchsanlagen belegt werden, auch bei höheren Durchlässigkeiten des Filtersubstrates. Bei den horizontalen Filtergräben wurde eine erhöhte Kolmationsgefahr festgestellt.

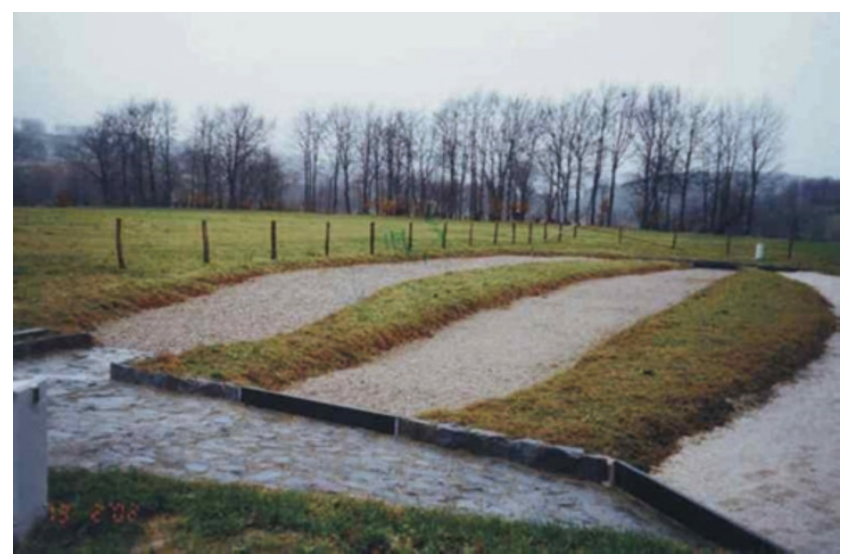

Abb. 3 Horizontale Filtergräben 